\title{
Testing a postselectional account of across-dimension switch costs
}

\author{
STEFANIE I. BECKER \\ University of Queensland, Brisbane, Queensland, Australia
}

\begin{abstract}
In visual search for a pop-out target, responses are faster when the target dimension from the previous trial is repeated than when it changes. Currently, it is unclear whether these across-dimension switch costs originate from processes that guide attention to the target or from later processes (e.g., target identification or response selection). The present study tested two critical predictions of a response-selection account of across-dimension switch costs - namely, (1) that switch costs should occur even when visual attention is guided by a completely different feature and (2) that changing the target dimension should affect the speed of responding, but not the speed of eye movements to the target. The results supported both predictions, indicating that changes of the target dimension do not affect early processes that guide attention to the target but, rather, affect later processes, which commence after the target has been selected.
\end{abstract}

From everyday experience, we all know that an object that looks very different from the surrounding objectsan orange among green apples, for example-appears to pop out from the background. Introspectively, we seem to be able to perceive such pop-out items immediately and without delay. However, research has shown that, in visual search for a pop-out target, responses are much faster when the target feature from the previous trial is repeated than when the target and nontarget features change from the previous trial (see, e.g., Maljkovic \& Nakayama, 1994). Currently, there is a debate about whether these intertrial effects, or switch costs, arise at an early or late level of processing. According to a visual-selection view, switch costs reside in early processes that guide visual attention to the target (e.g., Maljkovic \& Nakayama, 1994; Müller, Heller, \& Ziegler, 1995). By contrast, response-selection accounts assume that switch costs originate from later processes that commence after the target has been selected (e.g., Cohen \& Magen, 1999).

The question of whether intertrial switch costs occur at the level of visual selection or from later processes is important because switch costs signify that the processing of visual information is subject to certain limitations. If these limitations apply to the level of attention, it would, for instance, follow that pop-out items could not be perceived immediately and without delay.

\section{Feature Priming and Dimension Weighting}

Past research has shown that there are two different kinds of intertrial effects. First, feature priming effects are commonly found when the target and nontarget features directly swap on switch trials, so that the target inherits the features formerly associated with the nontargets and vice versa. Typically, feature priming effects are assessed in discrimination tasks, in which participants first search for a unique feature among several nontargets (e.g., shape) and then respond to a different feature of the target (e.g., the orientation of a bar inside the target; see the right panel of Figure 1 for an example).

The first studies showed that swapping the searchrelevant features of the target and the nontargets produces switch costs, whereas changing the response-relevant feature of the target across trials does not affect search performance. Maljkovic and Nakayama (1994) thus concluded that selection of the target biases attention toward the search-relevant feature of the target on the next trial(s), so that swapping the target and nontarget features delays visual selection of the target ( priming of pop-out hypothesis). Subsequent eye movement studies supported this view, by showing that swapping the search-relevant features led to more frequent selection of the nontargets and elongated the latencies of first eye movements to the target (Becker, 2008a, 2008b, 2010; Becker \& Horstmann, 2009; McPeek, Maljkovic, \& Nakayama, 1999; see also Goolsby \& Suzuki, 2001).

A different kind of intertrial effect, related specifically to changes of the target dimension, was found to occur in detection tasks, in which the target is present on half of all trials and observers have to indicate whether the target is present or absent. Importantly, only the target feature changes across trials, whereas the nontarget features remain constant. Analyses are usually restricted to target-present trials that are preceded by target-present trials, and performance is compared between two different conditions: (1) a within-dimension condition, in which 
Dimension Change

(detection task: present/absent)

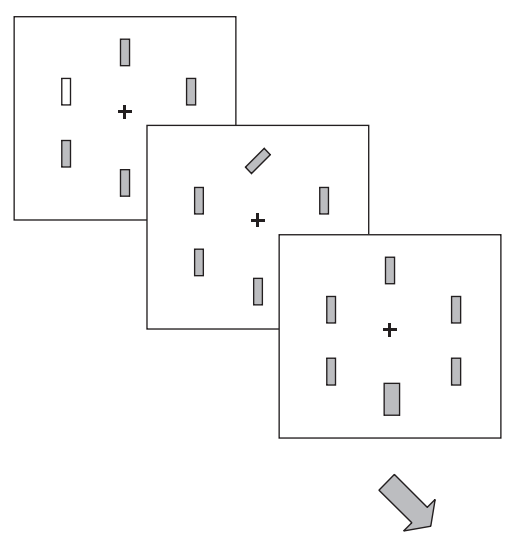

Feature Switch

(discrimination task: a vs. \)

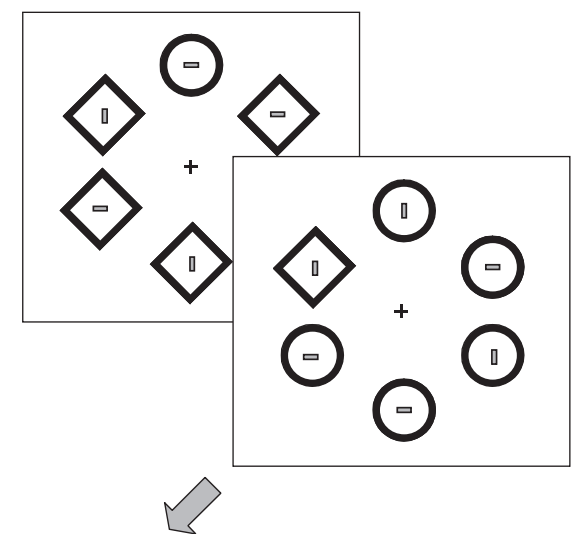

Combined: Search for shape/respond to dimension (discrimination task: $\square \diamond \square=$ right; $\square=$ left)
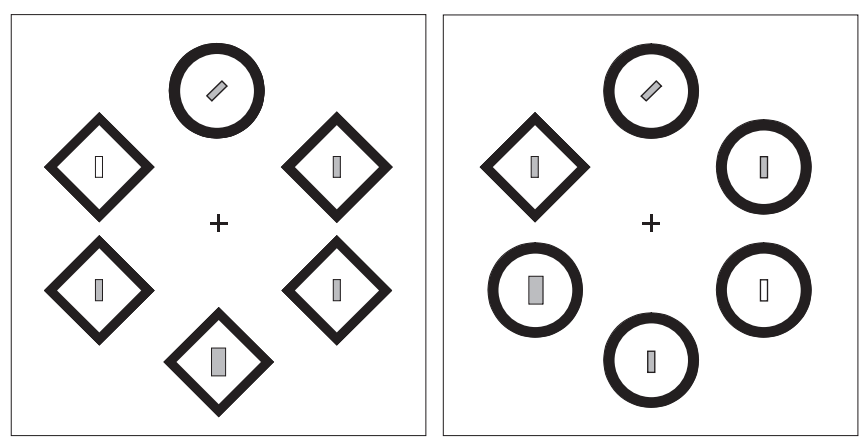

Figure 1. Example of the stimulus displays typically used to assess feature priming (top right panel) and across-dimension switch costs (top left panel). Experiment 1 used displays that combined characteristics from both paradigms (bottom panel): Participants had to search for a unique shape that randomly varied across trials, and to respond to a bar inside the target, which could be from different dimensions. The displays of Experiment 2 were identical to the classical displays used to assess across-dimension switch costs (top left panel).

only the target feature changes (e.g., between being tilted to the right or left, among vertical nontargets), and (2) an across-dimension condition, in which the target dimension changes across trials (e.g., between a green, tilted, or larger item, among gray, small, and vertical nontargets; see the left panel of Figure 1 for an illustration). The results typically show that changing the target feature in the within-dimension condition does not affect response times (RTs), whereas changes of the target dimension reliably produce switch costs on the order of 30-70 msec (e.g., Müller et al., 1995).

The finding that intertrial switch costs critically depended on changes of the target dimension is important because it indicates that, at some stage of visual processing, there are dimension-specific limitations but no feature-specific limitations. Despite the apparent inconsistency with feature priming effects, Müller et al. (1995) argued that dimension-specific switch costs occur at the level of visual selection, speculating that feature priming effects failed to emerge in the within-dimension condition because of differences between the tasks.
Contrary to such a visual-selection view of acrossdimension switch costs, it has been proposed that changing the target dimension does not predominantly affect the speed of visually selecting the target but impairs a different kind of process. Whereas some researchers have proposed that across-dimension switch costs originate from target identification processes (e.g., Becker, 2008a; Kumada, 2001; Theeuwes, Reimann, \& Mortier, 2006), others have suggested that they reside in later processes concerned with stimulus-to-response mappings or response selection (e.g., Cohen \& Magen, 1999; Mortier, Theeuwes, \& Starreveld, 2005). For the sake of simplicity, I will use the term postselectional view to collectively refer to these different views, which share the assumption that target dimension changes do not affect preattentive processes that guide attention to the target.

\section{Evidence for a Response-Selection Account of Across-Dimension Switch Costs}

To date, research has been dominated by the visualselection view of across-dimension switch costs, so that 
only three solid findings can be cited in support of the alternative, postselectional account: First, Kumada (2001) found that across-dimension switch costs were eliminated in a discrimination task, although this task presumably requires focal attention shifts to the target location, and argued that changing the target dimension does not interfere with focal attention shifts to the target, but with target identification. ${ }^{1}$ Second, Mortier et al. (2005) showed that across-dimension switch costs were not reduced or eliminated when a target or nontarget was presented alone and participants did not have to engage in search. These results indicate that changing the target dimension affects processes unrelated to search, contrary to a visualselection view of across-dimension switch costs. Third, an eye movement study by Becker (2008a) investigated the effects of different kinds of intertrial changes on the proportion of first saccades to the target and found that visual selection was impaired when the target and nontarget features directly swapped on switch trials, but not when only the target dimension or the target feature changed and the nontargets remained constant. Becker (2008a) proposed that switch costs at the level of visual selection occur only when the target and nontarget features swap, probably because observers are unable to tune attention toward targets with directly contradictory features (e.g., targets that are either larger or smaller than the nontargets; see also Becker, 2010). However, attention can apparently be tuned toward noncontradictory target features from different dimensions, allowing immediate visual selection when the feature or dimension of the target only changes.

\section{Defending a Visual-Selection View of Across-Dimension Switch Costs}

At first glance, the evidence against a visual-selection account of across-dimension switch costs may appear very convincing. However, a closer look reveals that much of the evidence is based on a null effect - that is, the failure to find across-dimension switch costs in discrimination tasks (e.g., Becker, 2008a, 2008b; Kumada, 2001). The absence of switch costs could, however, also be due to the fact that discrimination tasks require an additional attention shift, from the search-relevant feature to the dimension of the response-relevant item. This could, in turn, erase dimension-specific information from the previous trial, so that the absence of across-dimension switch costs is still compatible with a visual-selection view (cf. Müller \& Krummenacher, 2006). If this is correct, then the discrimination task would not be suitable for examining across-dimension switch costs.

Second, the finding that across-dimension switch costs persist even when the target is presented alone also does not present strong evidence against the visual-selection account. Given that the dimension of the target was still relevant to the response in the study, it is possible that participants tuned attention to the target dimension, although this was not necessary. Previous studies also indicate that attention can be tuned in parallel to the onset of the target and its particular feature. For instance, eye movements are often executed in the direction of an irrelevant onset distractor but change direction to the target in midflight, in- dicating that attention has been tuned both to the onset and to a specific feature of the target (see, e.g., Mulckhuyse, Van Zoest, \& Theeuwes, 2008; Theeuwes, Kramer, Hahn, \& Irwin, 1998). Thus, the currently available evidence is not sufficient to decide whether across-dimension switch costs are primarily due to early processes that guide attention to the target, or to later processes concerned with target identification or response selection (see also Müller \& Krummenacher, 2006).

\section{Aim of the Present Study}

The aim of the present study was to provide a more stringent test of the response-selection account of acrossdimension switch costs. The response-selection account makes some very strong predictions, none of which have been tested to date. First, the account implies that dimension change effects should occur even when the target has been selected by virtue of a completely different feature, and the dimension of the target is relevant only for the response. Second, the response-selection view predicts that repeating or changing the target dimension should affect the speed of responding, whereas it should not affect the speed of visually selecting the target. These predictions were tested in Experiments 1 and 2, respectively.

\section{EXPERIMENT 1}

The first experiment was designed to test the first critical prediction - that across-dimension switch costs should persist even when attention has been guided to the target by virtue of a different feature. To that aim, participants were instructed to make a fast eye movement to a unique shape in the display (search-relevant feature; circle or diamond), and to respond to a bar located inside the shape (responserelevant feature). The dimension of the response-relevant feature varied; in a manner similar to that of previous studies, participants had to respond with one button to small gray vertical items (corresponding to target-absent trials), and with another button to bars that were green, large, or tilted to the right (representing the target-present trials; see Figure 1, bottom panel). Deviating from previous studies, the target was, however, defined by its shape and could not be found by attending to dimension-specific information. To assess whether attention was indeed tuned to the target's shape, the shapes of the target and nontargets were randomly repeated or swapped across trials.

If attention and eye movements are guided to the target by virtue of its shape, swapping the shape of the target should lead to more frequent selection of the nontargets and should elongate the latencies of first saccades to the target (e.g., Becker, 2008a, 2008b; McPeek et al., 1999). Moreover, if across-dimension switch costs originate from postselectional processes, changing the dimension of the target should elongate RTs to the response-relevant feature, even though the item was selected by virtue of the surrounding shape.

\section{Method}

Participants. Nine volunteers, 2 male and 7 female (mean age, 29 years), were paid $\$ 10$ each for their participation in the experi- 
ment. All participants had normal or corrected-to-normal vision, and were naive as to the purpose of the experiment.

Apparatus. An Intel Core 2 Duo CPU 2.4-GHz computer with a 17-in. FP92E color monitor was used to run the experiment. Stimuli were presented with a resolution of $1,280 \times 1,024$ pixels and a refresh rate of $75 \mathrm{~Hz}$. Eye movements were tracked at $500 \mathrm{~Hz}$ with a video-based Eyelink 1000 tracker (SR Research, Ontario, Canada).

Stimuli, Design, and Procedure. The stimuli consisted of six black outlines of squares $\left(2.6^{\circ} \times 2.6^{\circ}\right)$ or circles (diameter, $\left.2.9^{\circ}\right)$ with a line width of $0.4^{\circ}$. The stimuli were positioned on the outlines of an imaginary circle (radius, $9.2^{\circ}$ ) and were presented against a white background. Observers were asked to make a fast eye movement to the odd shape and to respond to the item located in its center. Inside the six shapes, there were three gray small vertical items $\left(0.1^{\circ} \times 0.5^{\circ}\right)$, one green bar, a large gray bar $\left(0.3^{\circ} \times 0.6^{\circ}\right)$, and a gray bar tilted $45^{\circ}$ to the right. The target shape, target position, and the dimension of the response-relevant item were all chosen randomly on each trial.

To start the trial, observers had to fixate on the center of a central fixation cross for at least $500 \mathrm{msec}$ (within a time window of $2,000 \mathrm{msec}$ ). If the fixation control failed, participants were calibrated anew (9-point calibration). Otherwise, the search display was presented and remained on screen until the response. Participants were instructed to press the right mouse button when the target shape contained a small gray vertical item and to press the left mouse button for the green, tilted, and large bar. The search display was immediately followed by a $500-\mathrm{msec}$ feedback display consisting of the centrally presented words "correct" or "wrong" (Arial Black, 13-pt. black font). After an intertrial interval of $250 \mathrm{msec}$, in which a blank white screen was presented, the next trial started with the fixation control. The experiment consisted of 740 trials, and participants were encouraged to react both quickly and accurately.

\section{Results and Discussion}

Trials with RTs above 2,500 msec were excluded from all analyses (2.0\%), as were trials in which the target had not been selected - that is, when the gaze was not within 100 pixels $\left(2.3^{\circ}\right)$ of the center of the target (3.2\%). Data were statistically analyzed, using a $2 \times 2$ ANOVA comprising the variables " $n-1$ search-relevant feature" (same vs. different shape) and " $n-1$ response-relevant feature" (same vs. different dimension of the response-relevant feature). The most important comparison is between trials on which the stimulus dimension of the target is repeated versus changed without concomitant changes in the re- sponse button (i.e., trials with a large, tilted, or green response item that are also preceded by such trials).

A first analysis of the eye movement data in this condition confirmed that attention was guided to the target on the basis of its shape: The mean proportion of first saccades to the target was modulated by changes of the target shape, with $19 \%$ more saccades going directly to the target when the search-relevant feature was repeated than when it switched $\left[F(1,8)=22.87, M S_{\mathrm{e}}=146.9, p=.001, \eta^{2}=\right.$ .74]. By contrast, changes of the response dimension did not affect the precision of saccades (all $F_{\mathrm{s}}<1, p \mathrm{~s}>.36$ ), indicating that the target was indeed selected by virtue of its shape, and not by virtue of some combination of target shape and dimension of the response-related item. Since selection of a nontarget can conceivably lead to processing of the response-related item inside the nontarget, which can, in turn, interfere with carryover effects relating to the dimension of the response-related item, data were analyzed separately for trials on which the first eye movement went to the target as opposed to a nontarget (see the top and bottom panels of Table 1, respectively).

Only those trials on which the target was selected first showed mean RTs that were significantly slower when the target shape swapped than when it was repeated $[F(1,8)=$ $57.76, M S_{\mathrm{e}}=1,226.9, p<.001, \eta^{2}=.86$; see the top panel of Table 1]. More importantly, changing the dimension of the response-related item incurred significant switch costs, of $70 \mathrm{msec}$, when the target shape repeated, but not when it switched, leading to a significant interaction between shape priming and across-dimension switch costs $\left[F(1,8)=13.4, M S_{\mathrm{e}}=577.8, p=.006, \eta^{2}=.63\right]$. The mean error scores showed exactly the same nonsignificant trends (all $p \mathrm{~s}>.05$; see Table 1), indicating that the results were not due to a speed-accuracy trade-off.

The results indicate that across-dimension switch costs can occur even when attention is guided by a completely different feature, in support of a response-selection view. Interestingly, however, across-dimension switch costs were absent when the search-relevant shape swapped. This could be due to the fact that attention was covertly deployed to one of the nontargets prior to selection of

Table 1

Experiment 1: Effects of Repeating Versus Changing the Target-Defining Shape of the Target, and the Dimension of the Large, Green, or Vertical Response-Related Item, When the First Saccade Was Directed to the Target Versus a Nontarget

\begin{tabular}{|c|c|c|c|c|c|c|c|c|c|c|c|c|c|c|c|c|}
\hline \multirow[b]{3}{*}{ Shape } & \multicolumn{4}{|c|}{ RT (msec) } & \multicolumn{4}{|c|}{ Error $(\%)$} & \multicolumn{4}{|c|}{$\%$ First Saccade on Target } & \multicolumn{4}{|c|}{ Saccade Latency (msec) } \\
\hline & \multicolumn{2}{|c|}{ rep } & \multicolumn{2}{|c|}{ diff } & \multicolumn{2}{|c|}{ rep } & \multicolumn{2}{|c|}{ diff } & \multicolumn{2}{|c|}{ rep } & \multicolumn{2}{|c|}{ diff } & \multicolumn{2}{|c|}{ rep } & \multicolumn{2}{|c|}{ diff } \\
\hline & $M$ & SEM & $M$ & SEM & $M$ & SEM & $M$ & SEM & $M$ & SEM & $M$ & $S E M$ & $M$ & $\overline{S E M}$ & $M$ & SEM \\
\hline \multicolumn{17}{|c|}{ First Fixation on Target } \\
\hline Dim rep & 854 & 56.4 & 967 & 65.6 & 2.5 & 0.9 & 4.7 & 2.2 & 76.9 & 5.4 & 54.9 & 7.9 & 353 & 26.2 & 391 & 36.0 \\
\hline Dim diff & 925 & 65.5 & 979 & 66.4 & 7.8 & 2.5 & 6.4 & 1.5 & 75.8 & 4.3 & 59.1 & 7.2 & 367 & 32.7 & 400 & 38.7 \\
\hline Priming & $70^{*}$ & & 11 & & $5.4^{*}$ & & 1.6 & & -1.1 & & 4.2 & & 15 & & 10 & \\
\hline \multicolumn{17}{|c|}{ First Fixation on Nontarget } \\
\hline Dim rep & 1,264 & 121.4 & 1,341 & 89.4 & 7.4 & 5.5 & 9.2 & 3.7 & & & & & 345 & 41.8 & 350 & 33.1 \\
\hline Dim diff & 1,305 & 118.0 & 1,348 & 94.8 & 8.8 & 3.0 & 12.6 & 2.5 & & & & & 334 & 25.7 & 339 & 24.6 \\
\hline Priming & 41 & & 7 & & 1.4 & & 3.4 & & & & & & -11 & & -12 & \\
\hline
\end{tabular}

Note-Mean RTs were elevated when the dimension of the response-related item changed (between a large, green, and vertical item), indicating that across-dimension switch costs occur even when the target has been selected by virtue of its shape. Dim, dimension; rep, repetition of item; diff, change or switch of item. $\quad{ }^{*} p<.05$. 
Table 2

Experiment 1: Effects of Repeating Versus Switching the Search-Relevant Shape of the Target and the Dimension of the Small Gray and Vertical Response-Related Item, When the Shape Target Was Selected As the First Item

\begin{tabular}{|c|c|c|c|c|c|c|c|c|c|c|c|c|c|c|c|c|}
\hline \multirow[b]{3}{*}{ Shape } & \multicolumn{4}{|c|}{ RT (msec) } & \multicolumn{4}{|c|}{ Error $(\%)$} & \multicolumn{4}{|c|}{ \% First Saccade on Target } & \multicolumn{4}{|c|}{ Saccade Latency (msec) } \\
\hline & \multicolumn{2}{|c|}{ rep } & \multicolumn{2}{|c|}{ diff } & \multicolumn{2}{|c|}{ rep } & \multicolumn{2}{|c|}{ diff } & \multicolumn{2}{|c|}{ rep } & \multicolumn{2}{|c|}{ diff } & \multicolumn{2}{|c|}{ rep } & \multicolumn{2}{|c|}{ diff } \\
\hline & $M$ & $\overline{S E M}$ & $M$ & SEM & $M$ & $\overline{S E M}$ & $M$ & SEM & $M$ & SEM & $M$ & SEM & $M$ & SEM & $M$ & SEM \\
\hline Dim rep & 842 & 57.7 & 931 & 61 & 2.7 & 0.8 & 61 & 2.9 & 74.7 & 4.3 & 54.4 & 5.7 & 380 & 38.4 & 397 & 37.3 \\
\hline Dim diff & 913 & 58.4 & 998 & 73.4 & 6.8 & 1.8 & 7.1 & 2.3 & 71.8 & 5.3 & 59.1 & 5.3 & 376 & 36.1 & 402 & 38.2 \\
\hline Priming & $71^{*}$ & & $67^{*}$ & & 4.1 & & 1.0 & & -2.9 & & 4.7 & & -4 & & 5 & \\
\hline
\end{tabular}

Note-Changing from a large, green, or tilted item to a small gray vertical item incurred significant across-dimension switch costs, both when the target-defining shape was repeated and when it switched. Dim, dimension; rep, repetition of item; diff, change or switch of item. $\quad{ }^{*} p<.05$.

the target shape. This may have initiated processing of the response-relevant item inside the nontarget, thereby eliminating dimension-specific information from the previous trial. In line with this explanation, swapping the target shape elongated mean saccade latencies by $35 \mathrm{msec}$ $\left[F(1,8)=18.07, M S_{\mathrm{e}}=615.7, p=.003, \eta^{2}=.69\right]$, consistent with the view that covert attention was erroneously deployed to a nontarget.

Moreover, inspection of trials on which the eyes had selected a nontarget prior to the target showed that acrossdimension switch costs for shape repetition trials were reduced to $40 \mathrm{msec}$, and were nonsignificant $(F<1$; see the bottom panel of Table 1), in line with the view that selection of a nontarget can erase information from the previous trial.

However, it is still possible that across-dimension switch costs were absent on shape switch trials because the visual system was biased toward a switch in the response, which neutralized possible benefits from dimension repetition trials (e.g., Huang, Holcombe, \& Pashler, 2004; see also Becker, 2008b; Töllner, Grammar, Kiss, Müller, $\&$ Eimer, 2008). To assess whether such a response bias modulated RT, trials with the small gray vertical responserelated item were analyzed.

However, across-dimension switch costs for the small gray vertical item were significant both when the target shape was repeated $\left[F(1,8)=10.72, M S_{\mathrm{e}}=2,096.1\right.$, $\left.p=.011, \eta^{2}=.57\right]$ and when it switched $[F(1,8)=$ 9.96, $\left.M S_{\mathrm{e}}=2,043.5, p=.013, \eta^{2}=.55\right]$, leading to a significant main effect of dimension change $[F(1,8)=$ $102.19, M S_{\mathrm{e}}=418.7, p<.001, \eta^{2}=.93$ ] that was not modulated by feature priming $(F<1)$. This indicates that switching the shape did not produce a response bias to switch the response button, contrary to the response biasing account.

Taken together, the results provide strong evidence for a postselectional view of across-dimension switch costs. Previous research failed to reject a visual-selection view, because it could not rule out that the target was selected by virtue of its dimension, even when it was, for instance, presented alone (Mortier et al., 2005). The present results, however, indicate that across-dimension switch costs persist even when the item is selected by virtue of a completely different feature, thus providing strong evidence for a response-selection account of across-dimension switch costs.

\section{EXPERIMENT 2}

Experiment 2 tested the second critical prediction of a response-selection account, that changing the target dimension should not affect the speed of visually selecting the target, whereas it should delay manual responses to the target. Previous studies have shown that changing the target dimension did not produce switch costs in a discrimination task. However, if the task required an additional attention shift to the response-related item, it would be generally unsuitable for testing a visual-selection view (see, e.g., Müller \& Krummenacher, 2006). Experiment 2 avoided this complication by testing search performance in a present/absent detection task, where the target was a simple bar-without any shape outlines or additional response-related items - that was randomly large, green, or tilted and was presented among small gray vertical nontarget bars.

Performance was compared between a classical RT task, on which target presence or absence was reported by a buttonpress, and a saccade task, on which focal attention shifts were encouraged by asking participants to make a fast eye movement to the target (when it was present).

Both tasks used exactly the same stimulus conditions. Therefore, if switch costs in the RT task are due to delays in visually selecting the target, we would expect switch costs of the same magnitude in the saccade task. On the other hand, if across-dimension switch costs in the RT task are caused by processes that commence after visual selection of the target, we would expect across-dimension switch costs in the RT task, but not in the saccade task.

\section{Method}

Participants. Twenty-one new participants, 9 of them male (mean age, 25 years), participated in Experiment 2. Twelve of them participated in the saccade task and 9 in the RT task.

Apparatus, Stimuli, and Design. These were the same as in the previous experiment, with the following exceptions: The shape outlines were removed, so that the target-absent displays consisted of six small, gray vertical bars $(0.8 \times 2.8 \mathrm{~cm})$. On target-present trials, one of the bars was green, tilted $45^{\circ}$ to the left, or larger than the other bars $(1.35 \times 2.8 \mathrm{~cm}$; see Figure 1 , top left panel $)$. In the saccade task, participants had to make a fast eye movement to the unique item in the display when it was present, but they had to maintain fixation on the central fixation cross when the target was absent. A new trial was initiated by pressing the space bar. In the RT task, participants were instructed to maintain fixation during the trial and to press the right mouse button when the target was absent and the 
Table 3

Experiment 2: Across-Dimension Switch Costs in the Manual Response Time (RT) Task and Visual-Selection (Saccade) Task When the Target Could Be Randomly Tilted, Green, or Larger

\begin{tabular}{|c|c|c|c|c|c|c|c|c|}
\hline & \multirow{2}{*}{\multicolumn{4}{|c|}{ RT Task }} & \multicolumn{4}{|c|}{ Saccade Task } \\
\hline & & & & & \multirow{2}{*}{\multicolumn{2}{|c|}{$\begin{array}{c}\text { Saccade } \\
\text { Latency (msec) }\end{array}$}} & \multirow{2}{*}{\multicolumn{2}{|c|}{ Error (\%) }} \\
\hline & \multicolumn{2}{|c|}{ RT (msec) } & \multicolumn{2}{|c|}{ Error (\%) } & & & & \\
\hline & $M$ & $\overline{S E M}$ & $M$ & SEM & $M$ & $\overline{S E M}$ & $M$ & $S E M$ \\
\hline Dim rep & 630 & 26.7 & 2.5 & 1.0 & 353 & 20.1 & 7.2 & 1.1 \\
\hline Dim diff & 685 & 32.6 & 9.5 & 2.5 & 358 & 19.5 & 6.9 & 0.9 \\
\hline Priming & $55^{*}$ & & $7.0^{*}$ & & 5 & & -0.2 & \\
\hline
\end{tabular}

Note-The mean RT and error scores in the RT task show significant switch costs. By contrast, the speed and accuracy of saccades to the target were unaffected by changes of the target dimension, in line with a response-selection account of across-dimension switch costs. Dim, dimension; rep, repetition of item; diff, change or switch of item. ${ }^{*} p<.05$

left mouse button when the target was present. The target dimension varied randomly, with 50\% target-absent and target-present trials; participants completed 600 trials in each task.

\section{Results and Discussion}

Data were excluded from the analyses when the eyes had not been fixating on the target within $1,000 \mathrm{msec}$ from the onset of the trial in the saccade task $(<0.1 \%)$, or when RTs exceeded 1,500 msec in the RT task (3.8\%). The analyses included only target-present trials that were preceded by target-present trials. ${ }^{2}$

The results were clear-cut. In the RT task, changing the target dimension produced significant switch costs of $55 \mathrm{msec}$ in the mean RT $\left[F(1,11)=15.72, M S_{\mathrm{e}}=\right.$ 813.3, $p=.003, \eta^{2}=.68$; see Table 3], and observers also committed significantly more errors when the target dimension changed $\left[F(1,11)=13.84, M S_{\mathrm{e}}=16.3, p=\right.$ $\left..006, \eta^{2}=.63\right]$. Assessing across-dimension switch costs separately for each target type revealed significant switch costs between 49 and $70 \mathrm{msec}$ for all target types (green, tilted, large) and all intertrial changes (see Table 4).

By contrast, in the saccade task, changing the target dimension did not elongate the latencies of first saccades to the target $(F=1.9, p=.19)$ and did not did lead to more saccade errors $(F<1$; see Table 3$)$. Saccade errors

Table 4

Experiment 2: Across-Dimension Switch Costs in the Mean Response Time (RT) and Saccadic RT Tasks, Depicted Separately for Each Target Type and Intertrial Transition

\begin{tabular}{ccccccc}
\hline \multirow{2}{*}{$\begin{array}{c}\text { Current } \\
\text { Target Type }\end{array}$} & $\begin{array}{c}\text { Previous } \\
(n-1)\end{array}$ & $\begin{array}{c}c \\
\text { Target Type }\end{array}$ & $\begin{array}{c}\text { RT Task } \\
(\mathrm{msec})\end{array}$ & Priming & & \multicolumn{2}{c}{$\begin{array}{c}\text { Saccade Task } \\
(\mathrm{msec})\end{array}$} & Priming \\
\hline \multirow{2}{*}{ Tilted } & Tilted & 620 & - & & 362 & - \\
& Green & 673 & $53^{*}$ & & 362 & 0 \\
& Large & 690 & $70^{*}$ & & 349 & -12 \\
Green & Green & 594 & - & & 335 & - \\
& Tilted & 640 & $46^{*}$ & & 329 & -6 \\
\multirow{2}{*}{ Large } & Large & 653 & $59^{*}$ & & 338 & 3 \\
& Large & 664 & - & & 366 & - \\
& Tilted & 713 & $49^{*}$ & & 388 & $22^{*}$ \\
& Green & 738 & $74^{*}$ & & 391 & 25 \\
\hline
\end{tabular}

Note-In the manual RT task, all changes of the target dimension produced significant intertrial switch costs, whereas in the saccade task, acrossdimension switch costs were absent for all intertrial transitions, with the exception of the transition from a tilted to a large target. ${ }^{*} p<.05$. were also separately analyzed, according to whether (1) a nontarget had been selected prior to the target, (2) the eyes had not moved at all (misses), or (3) the eyes had failed to select the target. However, none of the error categories showed significant switch costs. ${ }^{3}$

Taken together, the results indicate that changing the dimension of the target affects response selection without affecting the speed of visually selecting the target, in line with a postselectional view of across-dimension switch costs.

However, saccade latencies were quite long, probably reflecting that observers adopted a rather conservative criterion for moving their eyes in the go/no-go task. To examine whether across-dimension switch costs could have been masked by long saccade latencies, the quartiles of the individual saccade latency distributions were computed, and latencies were averaged within the quartiles. Analysis of the binned saccade latencies showed that across-dimension switch costs were present only in the longest saccade latencies [last quartile, $F(1,10)=6.22$, $\left.M S_{\mathrm{e}}=381.0, p=.032, \eta^{2}=.38\right]$. Saccades with shorter latencies were unaffected by changes of the target dimension, resulting in a significant interaction between quartile and intertrial switch costs $[F(3,30)=6.79$, $\left.M S_{\mathrm{e}}=187.2, p=.014, \eta^{2}=.41\right]$. By contrast, the same analysis computed over the binned RTs in the RT task showed significant across-dimension switch costs across all quartiles of the distribution (all $F \mathrm{~s}>7.0$, all $p \mathrm{~s}<$ $.029)$, with no differences between quartiles $(F=1.79$, $p=.22$; see Table 5).

Contrary to the concerns raised above, the results show that the failure to detect switch costs in the mean saccade latencies was due to the fact that the majority of saccades were elicited too early to be subject to across-dimension switch costs. These results argue against a visual-selection view and instead support a postselectional view of acrossdimension switch costs.

\section{GENERAL DISCUSSION}

The present study critically tested and confirmed two important and hitherto untested predictions of a responseselection account of across-dimension switch costs. Experiment 1 showed that changing the dimension of an item 
Table 5

Experiment 2: Across-Dimension Switch Costs Depicted Separately According to Each Quartile of the Distribution of Response Times (RTs) in the RT Task and Saccade Latencies in the Saccade Task (in Milliseconds)

\begin{tabular}{lccccccc}
\hline & \multicolumn{3}{c}{ RT Task } & & \multicolumn{3}{c}{ Saccade Task } \\
\cline { 2 - 3 } \cline { 6 - 8 } Quartile & $\begin{array}{c}\text { Repeated } \\
\text { Item }\end{array}$ & $\begin{array}{c}\text { Different } \\
\text { Item }\end{array}$ & Priming & & $\begin{array}{c}\text { Repeated } \\
\text { Item }\end{array}$ & $\begin{array}{c}\text { Different } \\
\text { Item }\end{array}$ & Priming \\
\hline First & 505 & 544 & $39^{* *}$ & & 280 & 277 & -3 \\
Second & 567 & 618 & $51^{* *}$ & & 325 & 326 & 1 \\
Third & 634 & 687 & $53^{* *}$ & & 370 & 375 & 5 \\
Fourth & 819 & 888 & $69^{*}$ & & 442 & 463 & $21^{*}$ \\
\hline
\end{tabular}

Note-Across-dimension switch costs were significant across all quartiles of the RT distribution in the manual RT task, but were restricted to the last quartile of the saccade latency distribution in the saccade task. ${ }^{*} p<.05 . \quad{ }^{* *} p<.01$.

produced switch costs at the level of the manual response, even when attention had been guided toward the target by a completely different feature. Experiment 2 showed that the same conditions that caused large across-dimension switch costs at the level of the response did not affect the speed of eye movements: In the saccade task, only lateonset saccades were further delayed with changes of the target dimension, and these effects were considerably smaller $(20 \mathrm{msec})$ than the across-dimension switch costs of 50 msec observed in the RT task.

These findings, and especially the lack of acrossdimension switch costs in saccades that were elicited earlier, indicate that changes of the target dimension do not predominantly affect the time course of attention shifts that usually precede eye movements to a location (e.g., Deubel \& Schneider, 1996). Rather, changes of the target dimension appear to interfere with later processes that commence after attention has been deployed to the target.

However, the finding that dimension changes could interfere with late-onset saccades suggests that acrossdimension switch costs cannot completely reside in very late processes, concerned, for example, with response selection or response execution. Instead, at least a portion of across-dimension switch costs apparently originates from earlier processes that commence directly after attention has been shifted to a location and that may be concerned with target identification and/or stimulus-to-response mappings.

In the present study, changes of the target dimension may have affected late-onset saccades, because after the initial attention shift, the visual system proceeded to identify the covertly selected item before executing the saccade. Target identification may have been instigated prior to executing the saccade in order to gain certainty that the selected item was in fact the target (see Huang et al., 2004). Target identification would, however, have taken longer on different-dimension trials than on repetition trials, explaining why only late-onset saccades showed across-dimension switch costs.

The view that changes of the target dimension do not affect the speed of attention shifts to the target, but processes that commence directly afterward, is also in line with earlier findings - that changing the target dimension can elongate the mean dwell times on the target without affecting the speed of visually selecting the target (see, e.g., Becker, 2008b; but see also Töllner et al., 2008). If this interpretation is correct, it would argue against a strict version of an early selection account of across-dimension switch costs as well as a pure response-selection view, because switch costs would originate neither from very early perceptual processes that guide attention to the target nor from very late processes concerned with response selection or execution, but instead, from late perceptual and/ or early response-selection stages concerned with target identification and stimulus-to-response mappings.

Alternatively, the findings could be interpreted to show that changes of the target dimension can affect visualselection processes to a small degree, whereas the larger portion of across-dimension switch costs has to be explained by later processes (e.g., Theeuwes et al., 2006). However, this hybrid account seems to imply that there are "weak" dimension-specific limitations on visual selection and "hard" dimension-specific limitations on later processes. It is difficult to see how these limitations could be implemented. As a solution, Rangelov, Müller, and Zehetleitner (in press) recently proposed independent dimension weighting mechanisms for visual selection and target identification (see also Krummenacher, Grubert, \& Müller, 2010). However, the present results do not support the hybrid view, that there are weak dimension-specific limitations in visual selection, because the first percentiles of the saccade latency distribution did not show any evidence for switch costs. The hybrid account would also seem to offer a less parsimonious explanation than the idea that dimension changes usually affect target identification processes, which, however can interfere with visual selection of the target when the latter is delayed for some reason.

Attributing across-dimension switch costs to postselectional processes that commence shortly after target selection has some interesting implications: most importantly, perhaps, that visual selection and postselectional processes are based on processes that are subject to different limitations. In particular, visual selection is not subject to dimension-specific limitations, as can be seen in the absence of across-dimension switch costs in early-onset saccades. By contrast, manual responses to report the pres- 
ence or absence of a target in a detection task are subject to such dimension-specific limitations, as can be seen by the reliable occurrence of across-dimension switch costs. This dissociation is reminiscent of the difference between attention for perception and attention for action, which constitute different selection mechanisms that operate on different principles and are subject to different restrictions or limitations (e.g., Neumann \& Klotz, 1994). However, it is unclear whether the existence of two different attentional bottlenecks would be due to differences in the response modality (visual selection vs. response selection), or to differences between the task demands.

In line with the latter view, it is possible that the requirement to report the presence of the target draws on perceptual knowledge ("what knowledge") that requires entirely different processes than those that are necessary to indicate the target location ("where knowledge"; e.g., Kumada, 2001). However, the assumption that detection and visual-selection tasks are based on mutually exclusive processes seems to be at odds with the finding that changes of the target dimension can interfere with late-onset saccades (Experiment 1). Moreover, this view also appears to be inconsistent with the findings that switch costs can also occur in simple detection tasks, when the target and nontarget features directly swap (cf. Becker, 2008a; Kristjánsson, 2006). The latter finding indicates that visual selection (or "where knowledge") also plays a role in simple detection tasks, contrary to the notion that detection and discrimination tasks are based on mutually exclusive processes.

However, an important problem of the "standard" postselectional view is that some of its implications are slightly implausible: If we assume that visual selection of the target is generally completed at an early point in time and does not suffer from across-switch costs, then why are the target present/absent responses not based on information about the location of the pop-out target (e.g., saliency signals)? Why would observers delay their "target-present" response and instead try to identify the target when they must already know that the target is present? The inherent implausibility of this proposition has puzzled researchers for over a decade, and was instrumental in the adoption of a visual-selection view in the first place (see Found \& Müller, 1996; Müller et al., 1995).

However, a postselectional view of across-dimension switch costs may appear more plausible when we consider that visual selection may be so error prone that a mechanism initiating responses on the basis of location information (or saliency information) might actually harm performance more than it could benefit it. In addition, information about the target location may not constitute "knowledge" in a strict sense, because detecting an error in visual selection may require identification of the selected item (at least to a certain level). Viewed from this perspective, a mechanism that imposes stricter limitations on response selection than on visual selection appears to be very adaptive - especially when we assume that visual selection usually precedes and informs response selection. However, further research is required to investigate the limitations of visual selection and postselectional processes in more detail.

\section{AUTHOR NOTE}

The present research was supported by a UQ postdoctoral fellowship granted to S.I.B. I thank Will Harrison, Jess Irons, Hermann Müller, Tram Neill, Roger Remington, James Retell, and Takatsune Kumada for their insightful and helpful comments on earlier drafts of the manuscript. Correspondence concerning this article should be addressed to S. I. Becker, University of Queensland, School of Psychology, St Lucia, QLD 4072, Australia (e-mail: s.becker@psy.uq.edu.au).

\section{REFERENCES}

BeCKer, S. I. (2008a). Can intertrial effects of features and dimensions be explained by a single theory? Journal of Experimental Psychology: Human Perception \& Performance, 34, 1417-1440. doi:10.1037/ a0011386

BECKER, S. I. (2008b). The stage of priming: Are intertrial repetition effects attentional or decisional? Vision Research, 48, 1417-1440. doi:10.1016/j.visres.2007.10.025

BECKER, S. I. (2010). The role of target-distractor relationships in guiding attention and the eyes in visual search. Journal of Experimental Psychology: General, 139, 247-265. doi:10.1037/a0018808

BeCKER, S. I., \& HorstmanN, G. (2009). A feature-weighting account of priming in conjunction search. Attention, Perception, \& Psychophysics, 71, 258-272. doi:10.3758/APP.71.2.258

Cohen, A., \& MAgen, H. (1999). Intra- and cross-dimensional visual search for single-feature targets. Perception \& Psychophysics, 61, 291-307.

Deubel, H., \& SchneIder, W. X. (1996). Saccade target selection and object recognition: Evidence for a common attentional mechanism. Vision Research, 36, 1827-1837.

Found, A., \& MüLleR, H. J. (1996). Searching for unknown feature targets on more than one dimension: Investigating a "dimensionweighting" account. Perception \& Psychophysics, 58, 88-101.

Goolsby, B. A., \& SUZUKI, S. (2001). Understanding priming of colorsingleton search: Roles of attention at encoding and "retrieval." Perception \& Psychophysics, 63, 929-944.

Huang, L., Holcombe, A. O., \& Pashler, H. (2004). Repetition priming in visual search: Episodic retrieval, not feature priming. Memory \& Cognition, 32, 12-20.

KRISTJÁNSSON, A. (2006). Simultaneous priming along multiple feature dimensions in a visual search task. Vision Research, 46, 2554-2570.

Krummenacher, J., Grubert, A., \& MüLler, H. J. (2010). Inter-trial and redundant-signals effects in visual search and discrimination tasks: Separable pre-attentive and post-selective effects. Vision Research, 50, 1382-1395.

KumadA, T. (2001). Feature-based control of attention: Evidence for two forms of dimensions weighting. Perception \& Psychophysics, 63, 698-708

Maljkovic, V., \& Nakayama, K. (1994). Priming of pop-out: I. Role of features. Memory \& Cognition, 22, 657-672.

McPeek, R. M., Maljkovic, V., \& Nakayama, K. (1999). Saccades require focal attention and are facilitated by a short-term memory system. Vision Research, 39, 1555-1566.

Mortier, K., Theeuwes, J., \& Starreveld, P. (2005). Response selection modulates visual search within and across dimensions. Journal of Experimental Psychology: Human Perception \& Performance, 31, 542-557.

Mulckhuyse, M., Van Zoest, W., \& Theeuwes, J. (2008). Capture of the eyes by relevant and irrelevant onsets. Experimental Brain Research, 186, 225-235.

Müller, H. J., Heller, D., \& Ziegler, J. (1995). Visual search for singleton feature targets within and across feature dimensions. Perception \& Psychophysics, 57, 1-17.

Müller, H. J., \& Krummenacher, J. (2006). Locus of dimension weighting: Preattentive or postselective? Visual Cognition, 14, 490513.

Neumann, O., \& KLotz, W. (1994). Motor responses to nonreportable, masked stimuli: Where is the limit of direct parameter specification? In C. Umiltà \& M. Moscovitch (Eds.), Attention and performance $X V$ : Conscious and nonconscious information processing (pp. 123-150). Cambridge, MA: MIT Press.

Rangelov, D., Müller, H. J., \& Zehetleitner (in press). Indepen- 
dent dimension-weighting mechanisms for visual selection and target identification. Journal of Experimental Psychology: Human Perception \& Performance. doi: 10.1037/a0020364

Theeuwes, J., Kramer, A. F., Hahn, S., \& Irwin, D. E. (1998). Our eyes do not always go where we want them to go. Psychological Science, 9, 379-385.

Theeuwes, J., Reimann, B., \& Mortier, K. (2006). Visual search for featural singletons: No top-down modulation, only bottom-up priming. Visual Cognition, 14, 466-489.

Töllner, T., Gramann, K., Kiss, M., Müller, H. J., \& Eimer, M. (2008). Electrophysiological markers of visual dimension changes and response changes. Journal of Experimental Psychology: Human Perception \& Performance, 34, 531-542.

\section{NOTES}

1. Subsequent studies occasionally found across-dimension switch costs even in discrimination tasks (e.g., Müller \& Krummenacher, 2006; Töllner et al., 2008). However, switch costs in these studies amounted to only $10 \mathrm{msec}$ and, thus, do not seem to be large enough to explain the large across-dimension switch costs commonly found in detection tasks. Moreover, switching the stimulus dimension also interacted sig- nificantly with switching the response. These results would be consistent with the view that switch costs arise at the level of stimulus-to-response mappings and, thus, do not provide unambiguous evidence for a visualselection view.

2. In the RT task, target-absent trials showed significant switch costs $[t(8)=6.4, p<.001$; mean RT on repetition trials, 640 msec; switch trials, $719 \mathrm{msec}$ ], and these switch costs did not differ from switch costs on target-present trials. Despite this, data on target-absent trials were excluded because they are confounded with changes of the response (left/right).

3. Saccade errors resulting from selection of a nontarget and the failure to make any eye movements were committed more frequently on repetition trials than on switch trials $(0.4 \%$ nontarget selection on repetition trials vs. $0.0 \%$ on switch trials; $2.9 \%$ failure to move the eyes on repetition trials vs. $2.5 \%$ on switch trials). Only the failure to select the target prior to pressing the space bar (to initiate the next trial) showed a trend for switch costs, with $0.0 \%$ errors on repetition trials and $0.4 \%$ on switch trials, but none of the differences described above were significant (all $p \mathrm{~s}>.10)$.

(Manuscript received December 13, 2009; revision accepted for publication August 6, 2010.) 